\title{
Laparoscopic transperitoneal heminephrectomy for treatment of the nonfunctioning moiety of duplex kidney in adults: A case series
}

\author{
Manmeet Singh, Samarth Agarwal, Apul Goel, Manoj Kumar, Ashish Sharma, Siddharth Pandey, \\ Satynarayan Sankhwar \\ Department of Urology, King George's Medical University, Lucknow, Uttar Pradesh, India
}

Purpose: We report the results and experience of a tertiary care center in laparoscopic transperitoneal heminephrectomy for the treatment of a non-functioning upper pole moiety of duplex kidney in adults.

Materials and Methods: The key point of the technique included the placement of a 6-Fr ureteric catheter in the healthy ureter at the beginning of the procedure under fluoroscopic guidance cystoscopically. A standard laparoscopic 3- to 4-port placement was done after placing the patient in a 45 to 90 degrees lateral decubitus position. The upper pole was mobilized transperitoneally and transected using a harmonic scalpel.

Results: A total of 17 patients aged 19 to 44 years underwent laparoscopic upper pole heminephrectomy. All patients had a complete duplicated renal collecting system on the ipsilateral side. Three patients were found to have ureterocele. The average blood loss was minimal (50-150 mL) with a mean of $95 \mathrm{ml}$. None of the patients required any blood transfusions. The mean operative time was 220 minutes (range, 160-315 minutes). The average length of hospital stay was 3 days (range, 2-4 days). Only 2 patients had Clavien-Dindo grade 3a complications, which were managed by double J stenting and percutaneous aspiration, respectively. Conclusions: Laparoscopic heminephrectomy is playing a cornerstone role in the treatment of the non-functioning moiety of duplex kidneys. The procedure is safe, efficient and offers the typical preoperative and postoperative benefits of laparoscopic surgery. The rate of complications in adults is acceptable and is similar to that reported in pediatric patients.

Keywords: Adult; Congenital abnormalities; Kidney; Laparoscopy

This is an Open Access article distributed under the terms of the Creative Commons Attribution Non-Commercial License (http://creativecommons.org/licenses/by-nc/4.0) which permits unrestricted non-commercial use, distribution, and reproduction in any medium, provided the original work is properly cited.

\section{INTRODUCTION}

Since the advent of laparoscopic upper pole heminephrectomy for duplex kidney, which was first described by Jordan and Winslow in the pediatric population in 1993, urologists have tended to perform this surgery using the laparoscopic technique [1,2]. Duplex kidney is a rare entity with an overall prevalence of 1 in 125 . Twenty percent of cases are bilateral. Duplex kidney is almost twice as common in women as in men. The condition is usually associated

Received: 22 October, 2018 - Accepted: 14 February, 2019

Corresponding Author: Samarth Agarwal

Department of Urology, King George's Medical University, Chowk, Lucknow 226003, Uttar Pradesh, India

TEL: +91-9702288820, E-mail: rebellite@gmail.com

ORCID: https://orcid.org/0000-0003-1390-6488 
with ureteroceles, vesicoureteral reflux, and ectopic ureters and is often accompanied by a poorly functioning upper pole segment [3].

For management of duplex kidney, upper pole heminephrectomy with ureterectomy if the upper pole moiety is nonfunctioning or ureteropyelostomy/uretero-ureterostomy with ureteric common sheath reimplantation if a functioning moiety is present is the standard of care [4]. Because of the manifestation during childhood as urinary tract infections (UTIs), urinary incontinence, and voiding dysfunction, duplex kidney is usually diagnosed early. Routine use of antenatal ultrasonography for diagnosing pediatric problems has also led to the identification of asymptomatic cases. This results in prompt treatment with surgical intervention, leading to the resolution of most cases. Presentation in adulthood differs, with flank pain and recurrent UTIs being more common [5].

Published reports on laparoscopic heminephrectomy are scarce, and most are confined to the pediatric population. Owing to the lower incidences and limited indications of heminephrectomy, the cohort of cases in most reported series is also small [3-5]. The published reports on laparoscopic heminephrectomy in adults are rarer still, with only a few case series reported to date. A systemic review with cumulative analysis was carried out by Clement et al. [6], who reported only 7 such retrospective studies with a total of 66 patients.

We report the results and experience of a tertiary care center in laparoscopic transperitoneal upper pole heminephrectomy for the treatment of the nonfunctioning duplex moiety in adults.

\section{MATERIALS AND METHODS}

After approval of the Institutional Review Board of King George's Medical University (approval number: 0324/ ETHICS/RCELL18), a retrospective analysis was done of 17 patients who presented with either flank pain or recurrent UTIs or urinary incontinence and who underwent laparoscopic heminephrectomy and ureterectomy between 2010 and 2015 at King George's Medical University, Lucknow. Written informed consent was obtained from the patients.

\section{Patient characteristics}

A total of 7 men and 10 women aged 19 to 44 years underwent laparoscopic upper pole heminephrectomy for duplicated collecting systems. Of the total, 9 and 8 patients had a duplicated system on the right and left sides, respectively, while 1 patient had a bilateral duplicated system with the right kidney having a nonfunctioning upper pole.

Ipsilateral kidney stones were detected in 2 patients, who had a single stone (10-15 $\mathrm{mm}$ in size) in the lower pole that was managed with mini-percutaneous nephrolithotomy at a later date. Three patients had insignificant stones sized $3 \mathrm{~mm}$ and $5 \mathrm{~mm}$. All patients had ectopic ureters with a complete duplicated renal collecting system. Three patients were found to have ureterocele.

The main complaint on admission was urinary incontinence since childhood in 3 female patients, flank pain in 5 patients, and recurrent UTI in the remaining patients. Of the 9 patients who had a history of recurrent UTI, 3 presented with high fever and required long-term antibiotic prophylaxis.

Preoperative imaging evaluation was done by using ultrasonography, intravenous pyelogram, and computed tomography (CT)/magnetic resonance imaging with urogram. The upper pole in all patients was invariably hydronephrotic with thin parenchyma. Tc99m-diethylenetriaminepentaacetate (DTPA) scanning was done in all patients to confirm the nonfunctioning moiety. A voiding cytogram was carried out in 5 patients who were also found to have a dilated upper ureter in the preoperative imaging. However,

Table 1. Demographic characteristics

\begin{tabular}{lc}
\hline \multicolumn{1}{c}{ Variable } & Value \\
\hline Age (y) & $32(19-44)$ \\
Sex & \\
Male & 7 \\
Female & 10 \\
Operating side & \\
$\quad$ Right & 9 \\
$\quad$ Left & 8 \\
Chief symptoms & \\
$\quad$ Urinary incontinence & 3 \\
$\quad$ Flank pain & 5 \\
$\quad$ Recurrent urinary tract infection & 9 \\
Body mass index (kg/m ${ }^{2}$ ) & $28(24-32)$ \\
Cortical thickness of involved kidney & $5(3.5-7.4)$ \\
(nonfunctioning moiety) (mm) & \\
No. of patients having associated stones with nonfunctioning moiety \\
$\quad$ Male \\
$\quad$ Female \\
$\quad$ Total \\
No. of patients with vesicoureteric reflux (lower ureter) \\
$\quad$ Male \\
$\begin{array}{l}\text { Female } \\
\text { Total }\end{array}$ \\
\hline Values are prented as mean (range) \\
2 \\
\hline
\end{tabular}

Values are presented as mean (range) or number only. 
none of them were found to have vesicoureteric reflux in the upper ureter. All 5 of them had incidentally detected grade 1 vesicoureteric reflux in the ureter of the normally functioning lower pole moiety. The duration of hospital stay, estimated blood loss and need for blood transfusion, time required for surgery, and postoperative complications (based on Clavien-Dindo grading) were also documented for each patient.

Patients with UTI received antibiotic therapy according to the results of the urine bacterial culture and institutional antibiogram until the results of the repeated urine culture were sterile or found to have insignificant bacterial growth. No patients had a history of previous operations. The demographic profile and other characteristics of the patients are reported in Table 1.

\section{Surgical technique}

After the induction of general anesthesia, cystoscopy was carried out routinely in all patients and a 6-Fr ureteric catheter was placed into the normal ureter under fluoroscopic guidance after we performed retrograde pyelography. We inserted a Foley catheter and a nasogastric tube in each patient. We could identify both ureteric orifices of the duplex kidney. The patient with a bilateral duplex kidney (Fig. 1) had 4 ureteric orifices clearly visible in the bladder cystoscopically. In all cases, we found the orifice of the upper pole moiety medial and distal to the lower pole ureter. Thereafter, the patient was positioned in a 45 to 90 degrees lateral decubitus position.

Bowel preparation was done routinely in our patients. Peritoneal access was obtained by Hasan's technique at the umbilicus, and the abdomen was insufflated up to 12 to 15

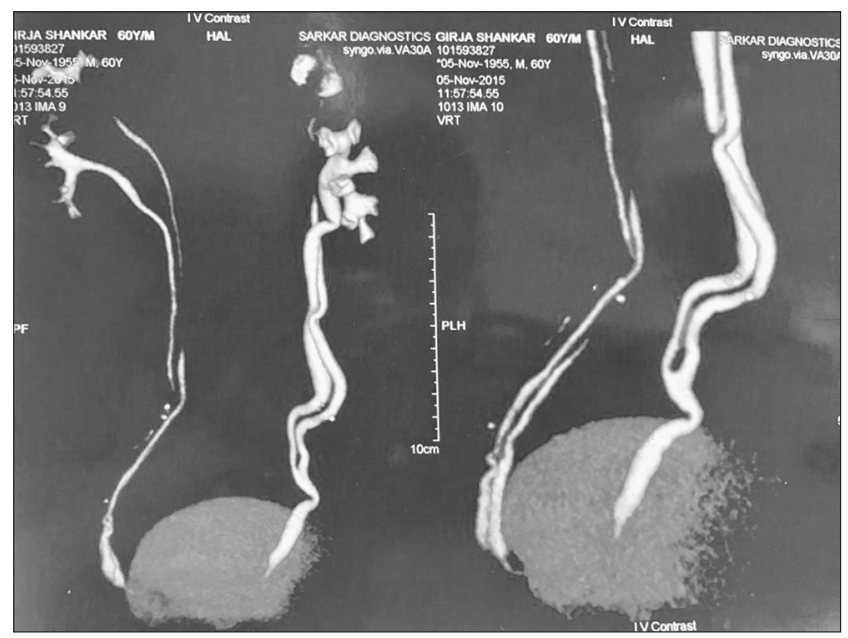

Fig. 1. Bilateral duplex system with bilateral ureters entering the posterior surface of the bladder. Note the poorly functioning upper pole moiety on the right side. $\mathrm{mm} \mathrm{Hg}$. All trocars were placed under direct vision. We used a 3-port technique on the left side and a 4-port technique on the right side for additional liver retraction. We used a $12-\mathrm{mm}$ camera port at the umbilicus, a $10-\mathrm{mm}$ port in the line joining the umbilicus and anterior superior iliac spine, and a third 5- or $10-\mathrm{mm}$ port subcostally completing the triangulation. The fourth port (5-mm) was placed in the midaxillary line when needed on the right side to retract the liver.

After incising the white line of Toldt and moving the colon medially, we identified the duplicated ureters medial to the lower pole. The Gerota fascia was incised. The normal lower pole ureter was identified by the 6 -Fr catheter that was previously placed. The diseased upper pole ureter was then dissected until the nonfunctioning hilum was reached. It is of paramount importance to take special care not to devascularize the blood supply to the lower ureter. To achieve such a meticulous dissection, we preferred using a combination of laparoscopic scissors and the harmonic scalpel and staying as close as possible to the serosa of the upper ureter. In most cases, we found vessels to the lower pole ureter crossing the upper ureter anteriorly. This again emphasized the fastidious need for dissection to prevent devascularization of the lower ureter while handling the upper ureter.

Once the nonfunctioning renal hilum was identified, the renal artery and vein were clipped using a Hem-olok ligation system, with 2 applied on each vessel. This

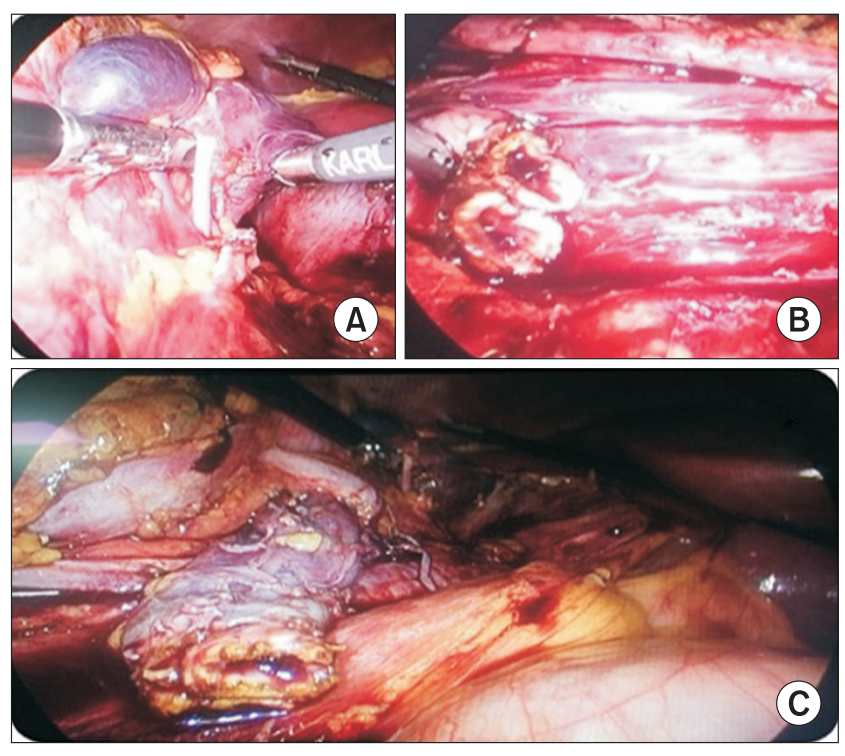

Fig. 2. (A) Intraoperative view showing identification and dissection of the upper pole nonfunctioning moiety of the right kidney. (B) This patient had a double ureter draining the nonfunctioning upper pole. (C) The nonfunctioning upper pole with double ureter clipped and dissected for removal. 
made it easier to demarcate the nonfunctioning upper pole parenchyma from the functioning one. Using electrocautery and the harmonic scalpel, we separated the upper pole from the rest of the renal parenchyma. We did not use any hemostatic agents like fibrin glue. Two patients had an opening of the calyces that was sutured intraoperatively with Vicryl 3-0. An abdominal drain was also placed in the postoperative period. In one case we identified that the upper pole moiety had double ureters (so in total 3 ureters) and so we had to dissect and clip both ureters as shown in Fig. 2.

\section{RESULTS}

The results are summarized in Table 2. All patients were adults. All patients underwent laparoscopic transperitoneal heminephrectomy, and no procedures were converted into open surgery. The average blood loss was minimal (range, $50-150 \mathrm{~mL}$ ); mean blood loss was $95 \mathrm{~mL}$. None of the patient required blood transfusion. The mean operating time was 220 minutes (range, 160-315 minutes). The longest time (315 minutes) was taken in a patient with a triplet ureter owing to difficulty in identification and dissection. The average length of hospital stay was 3 days (range, $2-4$ days). The nasogastric tube was removed on postoperative day 1 . All patients were discharged once they regained their bowel movements and passed flatus. The mean creatinine value in the postoperative period was 1.01 (range, 0.6-1.2) and did not differ significantly from preoperative creatinine values.

The drain was removed in most patients after 48 hours once the output had fallen to below $50 \mathrm{~mL}$. Two major complications were identified in the postoperative period. One patient showed extensive drainage continuously for 5 days in the postoperative period. On the 5th postoperative day, retrograde pyelography was performed and a leak was seen near the upper part of the ureter that was thought to be due to an iatrogenic injury sustained while the diseased ureter was being dissected. Consequently, the patient was stented with a 5-Fr double $\mathrm{J}$ stent, after which the output reduced dramatically.

The other patient also developed a Clavien-Dindo grade 3 complication. Although this patient was discharged on day 3 , she was readmitted on day 7 with sudden-onset, severe flank pain. A contrast-enhanced CT scan showed that she had a urinoma of around $300 \mathrm{~mL}$ in volume. It was drained percutaneously with ultrasound guidance under local infiltration. Although we initially contemplated placing a drain in this patient again, after aspiration her symptoms improved rapidly within 24 hours and she was completely pain-free after 48 hours. A repeat ultrasound after 48 hours showed no residual collection in the perinephric region and she was discharged.

All patients were followed-up on postoperative day 10 to 14 for suture removal. The mean period of follow-up was 8 months (range, 3-14 months).

The 6-Fr ureteral catheters were removed in the ope-

Table 2. Peri operative characteristics of the patients

\begin{tabular}{|c|c|c|c|c|c|c|c|c|}
\hline \multirow[b]{2}{*}{ No. } & \multirow[b]{2}{*}{ Sex } & \multirow{2}{*}{ Age (y) } & \multirow{2}{*}{$\begin{array}{l}\text { Time } \\
\text { (min) }\end{array}$} & \multirow{2}{*}{$\begin{array}{l}\text { Hospital stay } \\
\text { (d) }\end{array}$} & \multicolumn{2}{|c|}{ Creatinine (mg/dL) } & \multirow{2}{*}{$\begin{array}{c}\text { Postoperative } \\
\text { complications } \\
\text { (Clavien-Dindo) }\end{array}$} & \multirow[b]{2}{*}{ Management } \\
\hline & & & & & Preoperative & Postoperative & & \\
\hline 1 & Male & 44 & 300 & 4 & 1.1 & 1.2 & Grade 3a & 5-Fr double J stenting \\
\hline 2 & Female & 18 & 180 & 3 & 0.7 & 0.9 & - & - \\
\hline 3 & Female & 19 & 160 & 2 & 0.8 & 0.75 & - & - \\
\hline 4 & Male & 40 & 285 & 4 & 0.8 & 0.9 & - & - \\
\hline 5 & Female & 22 & 315 & 3 & 1.2 & 1.2 & Grade 3a & Percutaneous aspiration \\
\hline 6 & Male & 31 & 190 & 3 & 0.5 & 0.6 & - & - \\
\hline 7 & Female & 21 & 270 & 7 & 1.1 & 1.2 & - & - \\
\hline 8 & Female & 19 & 180 & 4 & 1.2 & 1.0 & - & - \\
\hline 9 & Male & 28 & 190 & 3 & 1.1 & 1.2 & - & - \\
\hline 10 & Female & 32 & 170 & 3 & 0.8 & 0.8 & - & - \\
\hline 11 & Female & 21 & 290 & 2 & 0.6 & 0.7 & - & - \\
\hline 12 & Female & 26 & 260 & 2 & 0.8 & 0.6 & - & - \\
\hline 13 & Male & 24 & 250 & 2.5 & 0.5 & 0.4 & - & - \\
\hline 14 & Female & 32 & 200 & 4 & 0.8 & 0.7 & - & - \\
\hline 15 & Male & 41 & 210 & 4 & 1.0 & 1.1 & - & - \\
\hline 16 & Male & 36 & 175 & 2 & 1.2 & 1.1 & - & - \\
\hline 17 & Female & 21 & 280 & 2 & 1.01 & 1.21 & - & - \\
\hline
\end{tabular}


rating room on completion of surgery. Histopathologic examination of the specimens revealed changes of chronic glomerulonephritis/pyelonephritis with chronic inflammatory cells and fibrosis.

\section{DISCUSSION}

Duplex kidney is a rare entity with an incidence of $0.8 \%$ [7]. The embryonic ureter develops from the ureteral bud, which itself develops as a branch from the mesonephric duct. As the bud grows and reaches the trigone, it gets absorbed, leaving the ureteric orifice near its normal position. In the case of duplication, the caudal ureter drains the lower pole and the cephalic drains the upper pole. The upper pole is more susceptible to obstruction because it is associated with ureteroceles or ectopic insertion and the lower pole has a greater association with vesicoureteric reflux [8].

Easier to diagnose and treat in children, duplex kidney often goes unnoticed in adults [9]. Most adult patients present with flank pain, hematuria, and UTIs [10]. In our series, recurrent UTIs were the most common presentation.

The surgical treatment for a nonfunctioning duplex moiety is heminephrectomy with removal of the diseased ureter. Since first reported by Jordan and Winslow in 1993 [11], several reports on the advantages and disadvantages of laparoscopic heminephrectomy have been published.

Most studies report heminephrectomy with either a transperitoneal or a retroperitoneal approach. We used a transperitoneal approach in our patients owing to the following advantages: a large working space with better manipulation of instruments, easy handling of the ureter, and best exposure of the hilum. The transperitoneal approach is also better in case of an infected system and complicated anatomical presentations, especially in duplicated systems [12,13].

Several complications have been reported, ranging from urinoma, recurrent UTIs, loss of a functioning lower pole, and prolonged urinary drainage [14]. Most studies report a varied complication rate of $6 \%$ to $50 \%$. Dönmez et al. [12] reported a complication rate of $50 \%$, as 5 patients developed major complications (Clavien-Dindo grade 3a and 3b). Similar to Dönmez et al. [12], Lee et al. [2] reported a complication rate of $6.3 \%$; in their study only 2 patients had complications that were deemed minor (Clavien-Dindo grade 1). Abedinzadeh et al. [15] reported their findings for 14 patients and had only 1 complication that resulted in functional loss of the remaining lower pole moiety. Abouassaly et al. [9] reported a complication rate of $20 \%$ with 1 of 5 surgical patients experiencing symptomatic urinoma at 1 week. Our study had a complication rate of $11.7 \%$ and both complications were Clavien-Dindo grade 3a. The higher complication rate could reflect the learning curve of the operative technique. The complication rate in pediatric published series is much lower, with most studies reporting rates of $2 \%$ to $8 \%$ because the procedures are commonly performed in the early years of life [16,17].

The mean operative time in our case series was 220 minutes with no requirement for blood transfusion. This was not much different from other reported studies. Abouassaly et al. [9] reported a mean operative time of 180 minutes. Lee et al. [2] reported the shortest mean operative time of 83 minutes. However, theirs was a case series of 32 patients. Mason et al. [18] reported a longer mean operative time of 310 minutes; theirs was a robotic approach and required extra time for docking the system in place.

Micturating cystogram was performed in 5 of our patients to rule out any associated vesicoureteric reflux as these patients also had a dilated upper pole ureter. However, none of the patients had vesicoureteric reflux in the upper ureter but all had incidentally detected grade 1 vesicoureteric reflux in the lower ureter. In the study reported by Abouassaly et al. [9], the authors mentioned the need to occlude the distal ureteric stump in case of refluxing nonfunctioning ureters and to leave the ureteric stump open in case of nonrefluxing ureters. This rules out the possibility of ureteral stump collections, refluxing diverticulum, and risk of pelvic abscess formation [18]

Several imaging modalities are available for diagnosis of nonfunctioning duplex kidneys. These include ultrasound, intravenous pyelogram, Tc99m DTPA scan, and CT. CT is particularly helpful in demonstrating hydronephrosis, thinning of the renal cortex, and uptake and excretion of contrast in the affected pole of the kidneys [19].

Our series demonstrates the effectiveness and low morbidity of the laparoscopic approach in the adult population. The existing literature regarding the present condition is sparse, especially in adults. We aim to increase understanding of the disease with better delineation of the surgical principles involved.

\section{CONCLUSIONS}

Laparoscopic heminephrectomy is now playing a cornerstone role in the treatment of the nonfunctioning moiety of duplex kidneys. It is safe and efficient and offers typical preoperative and postoperative benefits of laparoscopic surgery in adults with an acceptable rate of complications similar to that in pediatric patients. 


\section{CONFLICTS OF INTEREST}

The authors have nothing to disclose.

\section{ACKNOWLEDGMENTS}

I acknowledge the cooperation of residents of the urology department of King George's Medical University and the faculty and residents of the radiotherapy department who participated in data collection and evaluation of the patients. We also appreciate the commitment and compliance of the patients who reported the required data.

\section{REFERENCES}

1. El-Ghoneimi A, Farhat W, Bolduc S, Bagli D, McLorie G, Khoury A. Retroperitoneal laparoscopic vs open partial nephroureterectomy in children. BJU Int 2003;91:532-5.

2. Lee RS, Retik AB, Borer JG, Diamond DA, Peters CA. Pediatric retroperitoneal laparoscopic partial nephrectomy: comparison with an age matched cohort of open surgery. J Urol 2005;174:708-11; discussion 712.

3. Brehmer B, Makris A, Jakse G. Laparoscopic heminephrectomy and resection of ectopic ureter in an adult. Urol Int 2007;79:280-3.

4. Schlussel RN, Retik AB. Ectopic ureter, ureterocele, and other anomalies of the ureter. In: Walsh PC, Campbell MF, Retik AB. Campbell's urology. Philadelphia: Saunders; 2002;2007-52.

5. Janetschek G, Seibold J, Radmayr C, Bartsch G. Laparoscopic heminephroureterectomy in pediatric patients. J Urol 1997;158:1928-30.

6. Clement K, Hendry J, Keeley FX, Aboumarzouk OM. Heminephrectomy in adults: a systematic review with cumulative analysis. Urol Int 2018;101:1-6.

7. Malek RS, Kelalis PP, Stickler GB, Burke EC. Observations on ureteral ectopy in children. J Urol 1972;107:308-13.

8. Horst M, Smith GH. Pelvi-ureteric junction obstruction in duplex kidneys. BJU Int 2008;101:1580-4.

9. Abouassaly R, Gill IS, Kaouk JH. Laparoscopic upper pole partial nephrectomy for duplicated renal collecting systems in adult patients. Urology 2007;69:1202-5.

10. Gao Z, Wu J, Lin C, Men C. Transperitoneal laparoscopic heminephrectomy in duplex kidney: our initial experience. Urology 2011;77:231-6.

11. Jordan GH, Winslow BH. Laparoendoscopic upper pole partial nephrectomy with ureterectomy. J Urol 1993;150:940-3.

12. Dönmez MI, Yazici MS, Abat D, Kara Ö, Bayazit Y, Bilen CY. Laparoscopic upper pole heminephrectomy in adults for treatment of duplex kidneys. Urol J 2015;12:2074-7.

13. Yao D, Poppas DP. A clinical series of laparoscopic nephrectomy, nephroureterectomy and heminephroureterectomy in the pediatric population. J Urol 2000;163:1531-5.

14. Wang DS, Bird VG, Cooper CS, Austin JC, Winfield HN. Laparoscopic upper pole heminephrectomy for ectopic ureter: initial experience. Can J Urol 2004;11:2141-5.

15. Abedinzadeh M, Nouralizadeh A, Radfar MH, Moslemi MK. Transperitoneal laparoscopic heminephrectomy in duplex kidneys: a one centre experience. Ger Med Sci 2012;10:Doc05.

16. Leclair MD, Vidal I, Suply E, Podevin G, Héloury Y. Retroperitoneal laparoscopic heminephrectomy in duplex kidney in infants and children: a 15-year experience. Eur Urol 2009;56:3859.

17. Mushtaq I, Haleblian G. Laparoscopic heminephrectomy in infants and children: first 54 cases. J Pediatr Urol 2007;3:100-3.

18. Mason MD, Peters CA, Schenkman NS. Robot-assisted upper pole nephrectomy in adult patients with duplicated renal collecting systems. J Endourol 2012;26:838-42.

19. Zissin R, Apter S, Yaffe D, Kots E, Gayer G, Nissenkorn I, et al. Renal duplication with associated complications in adults: CT findings in 26 cases. Clin Radiol 2001;56:58-63. 